\title{
Importance of Phagosomal Functionality for Growth Restriction of Mycobacterium tuberculosis in Primary Human Macrophages
}

\author{
Amanda Welin $^{\mathrm{a}} \quad$ Johanna Raffetseder ${ }^{\mathrm{a}} \quad$ Daniel Eklund ${ }^{\mathrm{a}} \quad$ Olle Stendahl $^{\mathrm{a}}$ \\ Maria Lerm ${ }^{a}$ b \\ ${ }^{a}$ Medical Microbiology, Department of Clinical and Experimental Medicine, Faculty of Health Sciences, \\ Linköping University, Linköping, and ${ }^{b}$ Center for Infectious Medicine, Department of Medicine, Karolinska Institute, \\ Stockholm, Sweden
}

\author{
Key Words \\ Mycobacterium tuberculosis - Macrophages • Phagosomes • \\ Phagosomal maturation · Phagolysosomal fusion \\ lysosomes $\cdot$ Mycobacteria
}

\begin{abstract}
The best characterized survival mechanism of Mycobacterium tuberculosis inside the macrophage is the inhibition of phagosomal maturation. Phagosomal maturation involves several steps including fusion with lysosomes and acidification. However, it has not been elucidated which components of phagosomal maturation correlate with growth restriction of virulent mycobacteria in human macrophages, and we aimed to study this. We infected human monocyte-derived macrophages with $M$. tuberculosis and assessed bacterial replication, translocation of CD63 to the phagosome, and phagosomal acidification. We found that unstimulated human macrophages were able to control infection with $\mathrm{M}$. $\mathrm{tu}$ berculosis upon inoculation at a low multiplicity of infection (MOI) of 1, but not at a high $\mathrm{MOI}$ of 10 . The low $\mathrm{MOI}$ resulted in a macrophage-controlled balance between host cells and bacteria. Both H37Rv and H37Ra infection, at high and low $\mathrm{MOI}$, led to equally ineffective translocation of CD63 to the phagosome. On the other hand, acidification of mycobacte-
\end{abstract}

rial phagosomes was more efficient at $\mathrm{MOI} 1$ than 10 with both mycobacterial strains, consistent with a direct or indirect role for phagosomal acidification in restricting M. tuberculosis growth. Furthermore, inhibition of the vacuolar $\mathrm{H}^{+}-$ ATPase as well as of cathepsin D led to enhanced mycobacterial replication inside the macrophage. This again shows the importance of phagosomal acidification for control of mycobacterial growth, through the activation of lysosomal hydrolases. We conclude that acidification and related functional aspects of the mature phagosome are important factors for restriction of $M$. tuberculosis replication in human macrophages.

Copyright $\odot 2011$ S. Karger AG, Basel

\section{Introduction}

The causative agent of tuberculosis, Mycobacterium tuberculosis, primarily infects alveolar macrophages whose bactericidal mechanisms are modulated by the bacterium to create a niche suitable for survival and effective replication [1]. The best characterized survival mechanism of $M$. tuberculosis is that it inhibits maturation of the phagosome in which it resides in order to avoid lysosomal hydrolases and an acidic environment [2-5],

\section{KARGER \\ Fax +4161306 1234 \\ E-Mail karger@karger.ch}

www.karger.com
(C) 2011 S. Karger AG, Basel

1662-811X/11/0035-0508\$38.00/0

Accessible online at:

www.karger.com/jin
Dr. Amanda Welin

Medical Microbiology, Floor 12 Lab1

Department of Clinical and Experimental Medicine, Linköping University SE-58185 Linköping (Sweden)

Tel. +46 10103 2059, E-Mail amanda.welin@liu.se 
and another is escape from the phagosome into the cytosol [6]. These evasion mechanisms allow for replication of the bacilli and subsequent spread to other cells. However, it has not been fully elucidated which factor or factors determine the outcome of the infection - whether the bacteria are able to replicate intracellularly or whether the macrophage can control the infection. This question is crucial in understanding why only a small portion of infected individuals actually develop tuberculosis during their life time.

The fact that phagosomal maturation is partially inhibited by $M$. tuberculosis suggests that evasion of this mechanism is important for survival or replication of the bacillus $[7,8]$. M. tuberculosis protects itself from phagosomal acidification by preventing incorporation of the vacuolar $\mathrm{H}^{+}$-ATPase into the phagosomal membrane [811], and from phagolysosomal fusion by preventing delivery of lysosomal components to the phagosome [12]. Mycobacterial mutants defective in inhibition of phagosomal maturation display reduced survival within mouse macrophages [13], whereas macrophages activated with IFN- $\gamma$ or Toll-like receptor ligands have been shown capable of counteracting the bacterium's immunomodulating effects and restrict $M$. tuberculosis growth in some studies $[8,11,14,15]$. Nitric oxide production is crucial for growth restriction in mouse macrophages, while the mechanism in human macrophages is not as clear [14].

In this study, we investigated which components of phagosomal maturation correlate with inhibition of $M$. tuberculosis replication in unstimulated human macrophages, focusing on translocation of late endosomal/lysosomal proteins to the phagosome, phagosomal acidification and other phagosomal functions.

\section{Materials and Methods}

\section{Bacterial Strains and Growth Conditions}

The virulent $M$. tuberculosis strain H37Rv and the attenuated H37Ra were obtained from ATCC. $\gamma$-Irradiated M. tuberculosis was provided by Colorado State University, Fort Collins. For constitutive expression of green fluorescent protein (GFP), bacteria were transformed with the pFPV2 plasmid carrying the gene for GFP [16]. For selection of pFPV2-carrying mycobacteria, $20 \mu \mathrm{g} /$ $\mathrm{ml}$ kanamycin was used. For constitutive luciferase expression, bacteria were transformed with the pSMT1 plasmid carrying the lux $A B$ gene and selected using $100 \mu \mathrm{g} / \mathrm{ml}$ hygromycin. Early passages of $M$. tuberculosis were prepared and stored at $-70^{\circ} \mathrm{C}$. The bacteria were grown in Middlebrook $7 \mathrm{H} 9$ broth with $0.05 \%$ Tween-80, 0.5\% glycerol and ADC enrichment (Becton Dickinson) at $37^{\circ} \mathrm{C}$ for $2-3$ weeks, and then passaged and incubated for 7 days before use in experiments.

Phagosome Functionality and

M. tuberculosis Growth

\section{Macrophage Isolation}

Human monocytes were isolated from heparinized peripheral human blood and differentiated into human monocyte-derived macrophages (hMDMs) as reported previously by us $[17,18]$ and others $[19,20]$. Briefly, peripheral blood mononuclear cells were isolated and seeded in cell culture flasks, allowing selective enrichment of the adhesive monocytes and washout of lymphocytes. The monocytes were kept in Dulbecco's modified Eagle's medium with glucose (DMEM; Gibco; supplemented with $25 \mathrm{~mm}$ Hepes, $100 \mathrm{U} / \mathrm{ml}$ penicillin, $100 \mu \mathrm{g} / \mathrm{ml}$ streptomycin) and allowed to differentiate to hMDMs for 5-8 days in the presence of $10 \%$ active human serum pooled from 5 donors and $80 \mu \mathrm{M} \mathrm{L}$-glutamine. Determination of macrophage differentiation was based on adherence and phagocytic capacity. The day before the experiment, the hMDMs were trypsinized and re-seeded as required $\left(2.5 \times 10^{5}\right.$ per cover slip or $1 \times 10^{5}$ per well in 96-well plates), diluted in antibiotic-free medium with serum.

\section{Experimental Infection}

The bacterial suspension was centrifuged twice at $3,000 \mathrm{~g}$ for $10 \mathrm{~min}$, in phosphate-buffered saline (PBS) supplemented with $0.05 \%$ Tween- 80 , the pellet was resuspended in DMEM without additives and single bacilli were obtained by passaging the suspension $2 \times 10$ times through a sterile syringe equipped with a 27 -gauge needle. The concentration of living bacilli was determined by using optical density $\left(\mathrm{OD}_{600}\right)$ as a function of colonyforming units per milliliter, and microscopy confirmed efficient separation. Bacteria were added to the hMDMs at the indicated multiplicity of infection (MOI), diluted in DMEM without additives to achieve nonopsonic conditions. The hMDMs were incubated with the bacteria at $37^{\circ} \mathrm{C}$ for $1 \mathrm{~h}$ after which the medium was replaced with antibiotic-free DMEM with serum (pulsechase approach), before incubation for the indicated total times. Treatment of the hMDMs with bafilomycin A1 (Sigma-Aldrich; $100 \mathrm{nM}$ ) or diphenylene iodonium (DPI, $5 \mu \mathrm{M}$; Sigma-Aldrich) dissolved in DMSO (final concentration $0.1 \%$ ), or pepstatin A ( $1 \mu \mathrm{M}$; Roche) dissolved in methanol (final concentration $0.03 \%$ ) was carried out $1 \mathrm{~h}$ before infection, and the inhibitor was then included throughout the experiment.

For phagocytosis of zymosan, hMDMs were incubated with complement-opsonized and FITC-labeled zymosan particles (10:1) diluted in DMEM without additives for $1 \mathrm{~h}$, after which the medium was changed as described above. Infection with a GFPexpressing strain of Salmonella Typhimurium was performed in the same manner.

\section{Measurement of Intracellular Bacterial Growth}

Bacterial growth inside hMDMs over several days was assessed using a luminometry-based method, as previously described [18]. Briefly, hMDMs seeded in triplicate in 96-well plates were infected as described above with luciferase-expressing $\mathrm{H} 37 \mathrm{Rv}$, and hypotonically lysed at the indicated time points before addition of substrate ( $1 \%$ decanal) and measurement of luminescence using a Modulus microplate reader (Turner Biosystems). As previously validated, the amount of luminescence corresponded to the number of viable bacilli in each sample. The median value of each triplicate was used, and the amount of luminescence in the cell lysate at each time point was normalized against the phagocytosis value of each treatment $(4 \mathrm{~h})$ to account for donor variability in uptake. 


\section{Determination of Macrophage Viability}

For microscopic determination of hMDM viability, cells were incubated with Fixable Viability Dye eFluor 660 (eBioscience), diluted $1: 1,000$ in $\mathrm{PBS}$, at $4^{\circ} \mathrm{C}$ for $30 \mathrm{~min}$ prior to PFA fixation and subsequent immunofluorescence staining. The dye stains the nuclei of cells with damaged plasma membranes, allowing discrimination of live and compromised cells.

The relative number of viable hMDMs was assessed throughout the infection by a calcein acetoxymethyl (calcein-AM)-based method, as previously described [18]. Briefly, hMDMs seeded in black 96-well plates were infected, incubated for up to 7 days, washed with PBS and incubated with $4 \mu \mathrm{M}$ calcein-AM (Molecular Probes) at room temperature (RT) for $45 \mathrm{~min}$. As metabolically active cells convert calcein-AM to highly fluorescent calcein, the resulting fluorescence corresponds to the number of viable cells. Fluorescence was measured in a Modulus microplate reader using a 490-nm filter, and the values were normalized against the value for uninfected hMDMs at each time point to obtain the relative proportion of live cells.

\section{Immunofluorescence and F-Actin Staining}

Infected hMDMs on cover slips were fixed in $4 \%$ paraformaldehyde at RT for 30 min and washed 3 times. Staining was then performed in a humid chamber. The fixed cells were blocked and permeabilized in a solution containing PBS with 2\% BSA and $10 \%$ normal goat serum (Sigma-Aldrich), supplemented with $0.1 \%$ saponin (w/v) at RT for $30 \mathrm{~min}$. After 3 washes in PBS, the cover slips were incubated with either mouse monoclonal anti-LAMP-1 antibody (4 $\mu \mathrm{g} / \mathrm{ml}$; Santa Cruz) or mouse monoclonal anti-CD63 antibody (4 $\mu \mathrm{g} / \mathrm{ml}$; Sanquin) diluted in blocking solution at RT for $1 \mathrm{~h}$. After 35 -min washes, the cells were incubated with Alexa 594-conjugated goat anti-mouse IgG ( $5 \mu \mathrm{g} / \mathrm{ml}$; Molecular Probes), also diluted in blocking solution, at $37^{\circ} \mathrm{C}$ for $30 \mathrm{~min}$. Cover slips were washed and mounted using fluorescence mounting medium (Dako) and the preparations were re-coded to achieve unbiased analysis. For staining of F-actin, the hMDMs were permeabilized with $0.1 \%$ saponin (w/v) after fixation and incubated with $5 \mathrm{U} / \mathrm{ml}$ Alexa 594-coupled Phalloidin (Molecular Probes), diluted in PBS, for $30 \mathrm{~min}$ at RT, followed by washing and mounting in the same manner.

\section{LysoTracker Assay}

hMDMs on cover slips were infected and acidified compartments were labeled using LysoTracker Red DND-99 (75 nM; Molecular Probes) [21], which was added during the final $2 \mathrm{~h}$ of incubation, diluted in antibiotic-free DMEM with serum. The hMDMs were washed, fixed and mounted for confocal microscopy analysis, and images were obtained and evaluated in a blinded fashion.

\section{Microscopy}

Images were obtained using a confocal microscope (BioRad Radiance 2000 MP with LaserSharp 2000 software), with an argon laser emitting dually at $488 \mathrm{~nm}$ for excitation of FITC or GFP and at $514 \mathrm{~nm}$ for Alexa 594, and a red diode laser for excitation of eFluor 660. The samples were blinded throughout image acquisition and all analyses, after which the collected data were de-coded. Where indicated, a fluorescence microscope (Nikon Eclipse E800) was used.
Statistical Analysis

Paired Student's t test was used for statistical analysis, and $\mathrm{p}$ values of $\leq 0.05$ were considered significant.

\section{Results}

\section{hMDMs Are Able to Control Infection with H37Rv at} a Low MOI

In order to study what conditions are necessary for growth restriction of mycobacteria, we developed an infection model where unstimulated hMDMs were able to control infection with virulent $M$. tuberculosis. It is conceivable that the hMDMs are better equipped to deal with a low bacterial burden than a high one, so we evaluated the use of a low-MOI infection model to obtain a balance between the bacteria and the infected cells. hMDMs were infected with luciferase-expressing H37Rv at MOI 10 and 1 , and light emission, which was a correlate of the number of colony-forming units in the hMDM lysates, was measured over a time period of 1 week. Inoculation of the macrophages at MOI 10 resulted in effective bacterial replication, while inoculation at MOI 1 resulted in a balance between the macrophages and the bacilli, where very little replication occurred after the first few days of infection (fig. 1a). A calcein AM-based cell viability assay showed that at MOI 1, the macrophages remained viable throughout the experiment (typically $80-100 \%$ were viable after 1, 3 and 7 days, as compared to uninfected cells), while at MOI 10 the hMDMs were rapidly killed (typically less than $40 \%$ were viable after 1 day and less than $20 \%$ after 3 days, as compared to uninfected cells). After $4 \mathrm{~h}$, however, there was no significant difference between the number of viable hMDMs in uninfected samples and after infection with $\mathrm{H} 37 \mathrm{Rv}$ at MOI 10 or 1 . The same assay showed that upon infection of hMDMs with H37Ra at MOI 10, the majority (typically 70-90\%) of the macrophages were still viable after 3 days.

It is known that $\mathrm{H} 37 \mathrm{Ra}$ does not replicate inside macrophages [22], and we previously corroborated this finding in our macrophage model [18]. We confirmed this result by enumerating the number of GFP-expressing $\mathrm{H} 37 \mathrm{Rv}$ and H37Ra inside fixed hMDMs over 2 days of infection, after inoculation at an MOI of 10 (fig. 1b). Fluorescence microscopy images of hMDMs infected with $\mathrm{H} 37 \mathrm{Rv}$ at MOI 10 for up to 2 days, and stained for F-actin, show increased numbers of intracellular bacteria growing in clusters (fig. 1c). The luciferase assay showed that inoculation of DMEM (without antibiotics but with serum) with $\mathrm{H} 37 \mathrm{Rv}$ or $\mathrm{H} 37 \mathrm{Ra}$ in the absence of hMDMs, 


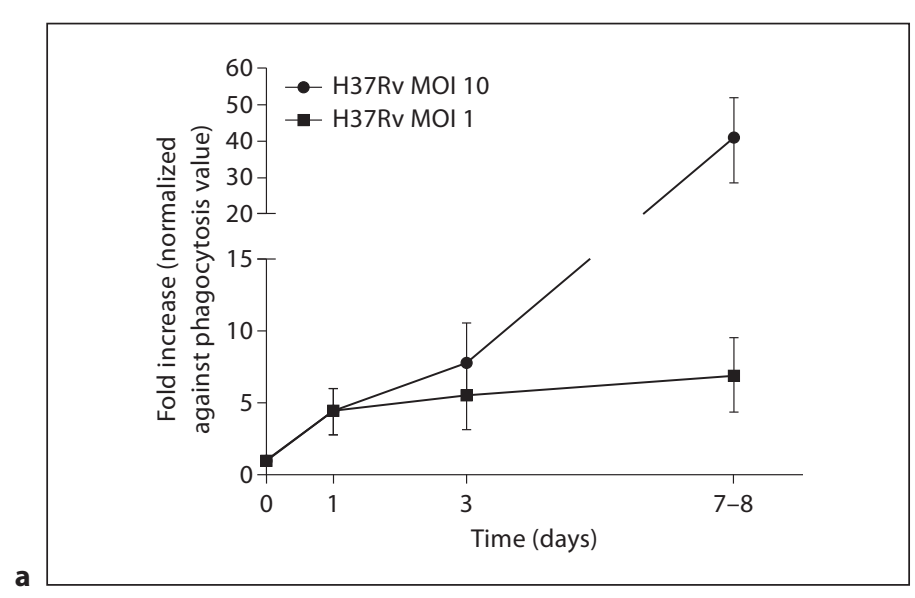

Day 0
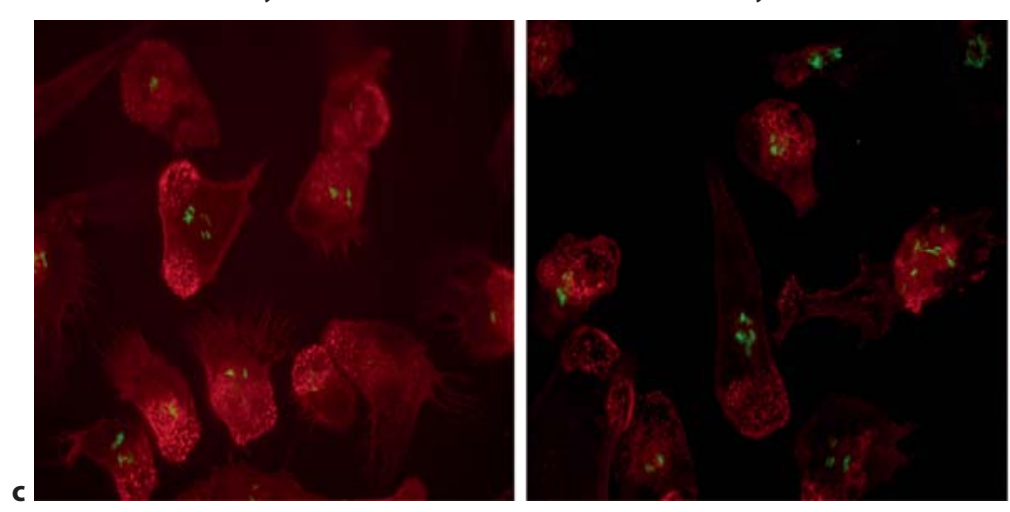

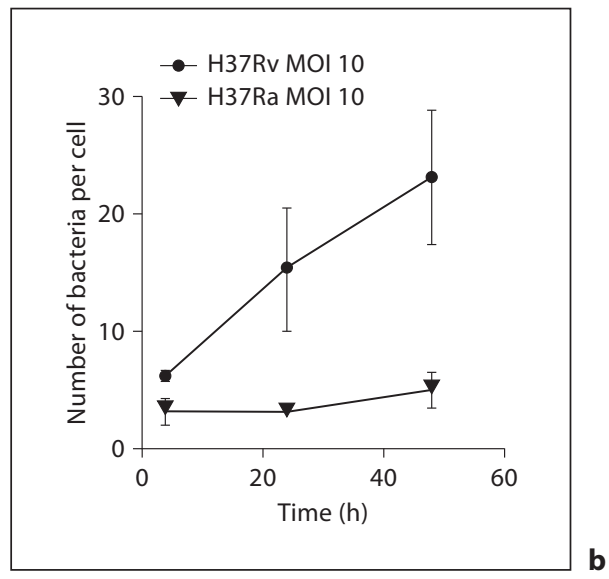

Day 2
Fig. 1. Intracellular replication of $\mathrm{H} 37 \mathrm{Rv}$ and $\mathrm{H} 37 \mathrm{Ra}$. hMDMs were infected at different MOIs with $\mathrm{H} 37 \mathrm{Rv}$ or $\mathrm{H} 37 \mathrm{Ra}$ for the indicated times, and the number of intracellular bacteria was analyzed. a Growth curve of luciferase-expressing H37Rv infecting hMDMs at MOI 10 or 1 . The relative number of viable intracellular bacilli was measured at each time point. The values were normalized against the phagocytosis value of each MOI. The figure shows the mean and SEM from 3 independent experiments. b Growth curve showing the mean number of bacilli per hMDM over 2 days of infection with GFP-expressing H37Rv or H37Ra at MOI 10. Enumeration of GFP-expressing bacteria was performed in 20 blinded images from each time point and donor. Means and SEM from 3 independent experiments are shown. c Representative fluorescence microscopy images of F-actin-stained (red) hMDMs infected with GFP-expressing H37Rv (green) at MOI 10 over 2 days. The images are merges of the green and red channels. or in the presence of pre-lysed hMDMs, resulted in effective growth of both strains of bacteria (data not shown). However, the same assay showed that the net increase in bacterial numbers inside hMDMs infected with H37Rv at MOI 10 was not due to extracellular growth and continuous phagocytosis because the addition of cytochala$\sin \mathrm{B}$, which inhibits actin polymerization and thus phagocytosis, did not affect $\mathrm{H} 37 \mathrm{Rv}$ growth in the intracellular fraction (data not shown). Our data thus indicate that unstimulated macrophages are able to inhibit growth of $\mathrm{H} 37 \mathrm{Rv}$ when a low MOI is used, and of H37Ra. This observation was used in the subsequent experiments to study what components of phagosomal maturation were important for the control of intracellular mycobacterial replication.

Growth Restriction at MOI 1 Does Not Correlate with

\section{CD63 Acquisition}

The first component of phagosomal maturation that we studied was the translocation of lysosomal-associated membrane proteins (LAMPs) to mycobacterial phagosomes. LAMPs such as CD63 (LAMP-3) are often used as an indicator of phagolysosomal fusion, although they are not found exclusively on lysosomes, but also on intermediate/late endosomes, and their usefulness in the context of mycobacterial phagosomes has been questioned $[8,23$, 
Zymosan
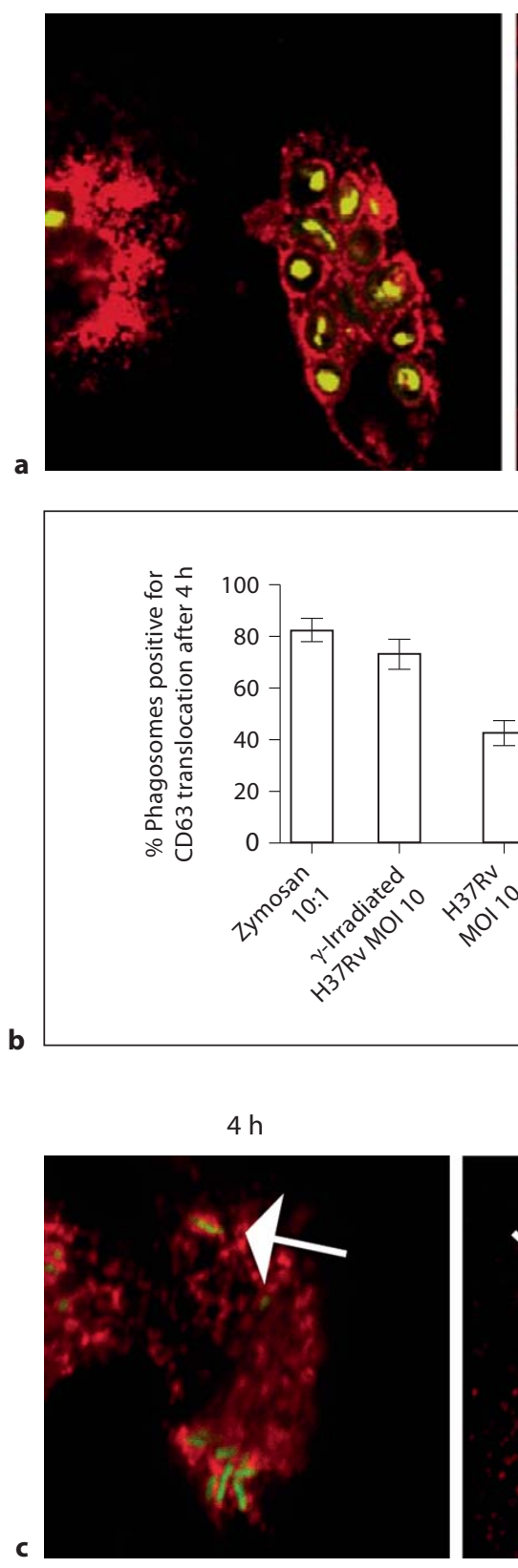

$4 \mathrm{~h}$

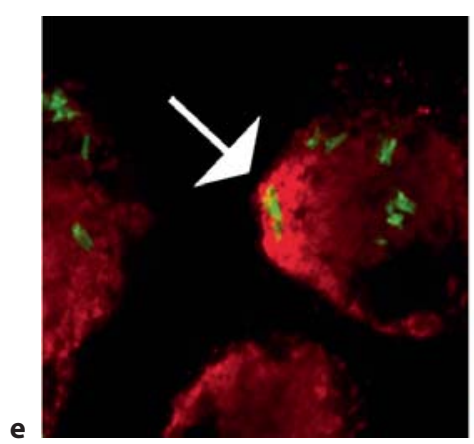

$48 \mathrm{~h}$

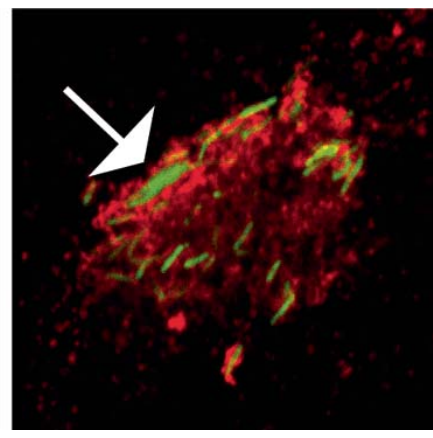

$48 \mathrm{~h}$

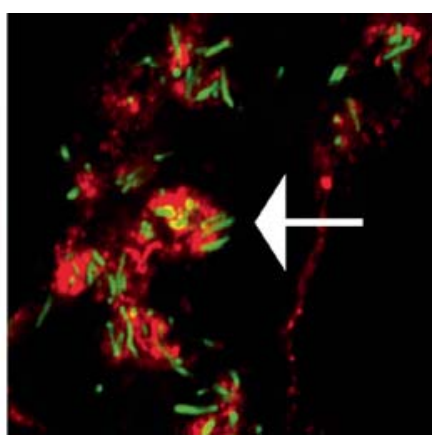

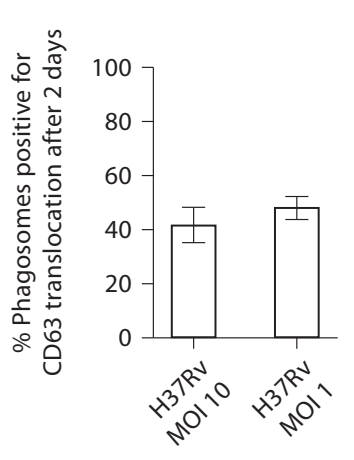
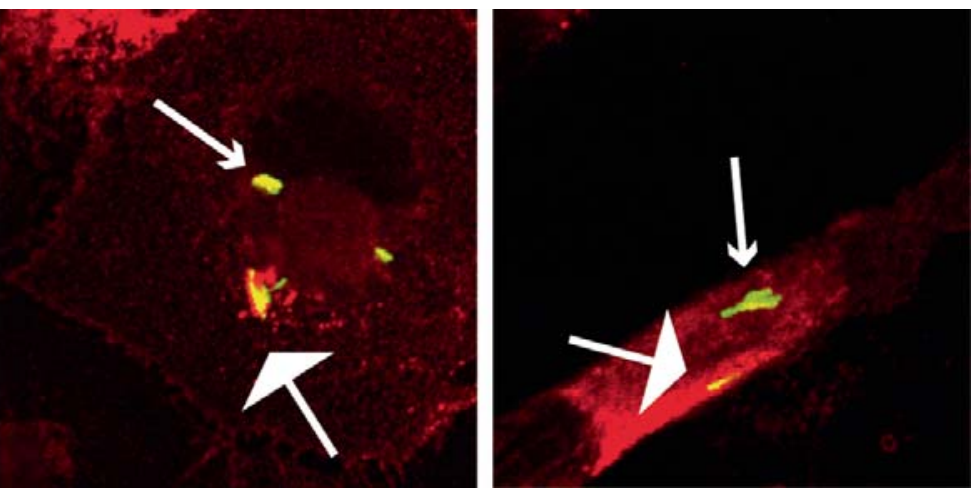

H37Ra

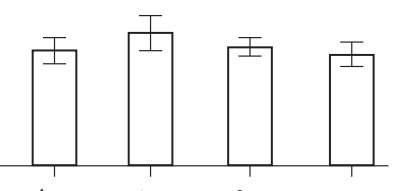

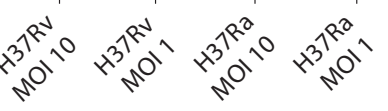
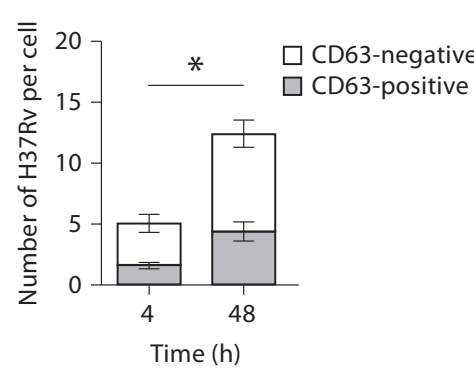

d

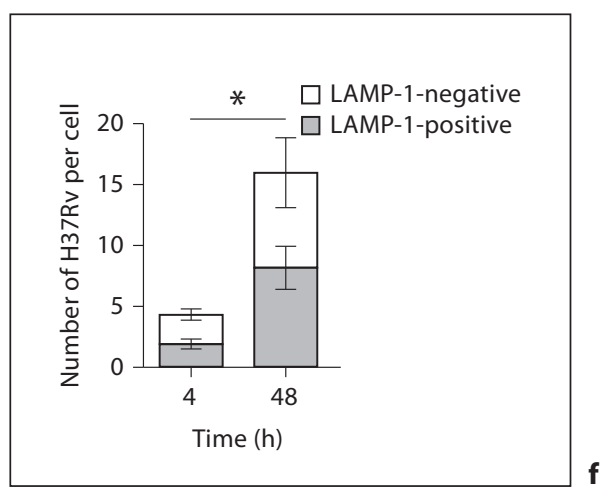


24]. In order to investigate to what extent macrophages are able to translocate CD63 to the mycobacterium-containing phagosome, immunostaining for CD63 was performed in H37Rv- or H37Ra-infected hMDMs. For comparison, phagosomes carrying dead H37Rv or zymosan particles were studied. The images of the H37Rv-, H37 Raand zymosan-challenged hMDMs showed phagosomes that were either positive or negative for CD63 staining (fig. 2a). To quantify the translocation of CD63 to the phagosome in macrophages infected with the different prey, the association of CD63 to phagosomes was analyzed in the confocal images. In this assay, single bacilli and aggregates were both scored as one phagosome event. As mentioned, extensive cell death occurred in hMDM cultures infected with $\mathrm{H} 37 \mathrm{Rv}$ at MOI 10, and these experiments thus included both viable and unviable macrophages. However, a control experiment where hMDMs were stained with a fixable fluorescent viability dye be-

Fig. 2. CD63 translocation to the phagosome after infection with different strains and MOI of M. tuberculosis. hMDMs were challenged with GFP-expressing live or $\gamma$-irradiated $\mathrm{H} 37 \mathrm{Rv}$, live H37Ra or FITC-labeled zymosan for the indicated times, and the late endosomal/lysosomal markers CD63 or LAMP-1 were immunostained. a Confocal images showing representative CD63stained (red) zymosan-, H37Rv- and H37Ra (green)-containing hMDMs after $4 \mathrm{~h}$ of infection. The images are merges of the green and red channels. The large arrows indicate phagosomes positive for CD63 transloca-tion, and the small arrows show phagosomes negative for CD63. $\mathbf{b}$ hMDMs challenged with live or $\gamma$-irradiated H37Rv or H37Ra at MOI 10 or 1 or zymosan at 10:1 were fixed after $4 \mathrm{~h}$ or 2 days, and CD63 was stained. The histograms show the mean percentage of phagosomes that were positive for CD63 (blinded evaluation of $>40$ phagosomes per preparation), and the SEM from at least 4 independent experiments. c hMDMs infected with $\mathrm{H} 37 \mathrm{Rv}$ (green) at MOI 10 for $4 \mathrm{~h}$ or $48 \mathrm{~h}$ were stained for CD63 (red). The confocal images show representative merges of GFP and CD63 after $4 \mathrm{~h}$ and $48 \mathrm{~h}$. The arrows indicate CD63-positive phagosomes. $\mathbf{d}$ The number of bacilli in CD63-positive and CD63negative compartments and the number of hMDMs were enumerated in CD63-stained hMDMs (blinded evaluation of $\geq 25$ hMDMs per preparation). The mean number of bacilli per macrophage that were positive and negative for CD63 after 4 and $48 \mathrm{~h}$ is shown. Error bars show SEM. ${ }^{*} \mathrm{p}<0.05$. e hMDMs infected with $\mathrm{H} 37 \mathrm{Rv}$ (green) at MOI 10 for 4 or $48 \mathrm{~h}$ from 6 independent experiments were stained for LAMP-1 (red). The confocal images show representative merges of GFP and LAMP-1 after 4 and $48 \mathrm{~h}$. The arrows indicate LAMP-1-positive phagosomes. $\mathbf{f}$ The number of bacilli in LAMP-1-positive and LAMP-1-negative compartments and the number of hMDMs were enumerated in CD63-stained hMDMs (blinded evaluation of $\geq 25 \mathrm{hMDMs}$ per preparation). The mean number of bacilli per hMDM that were positive and negative for LAMP-1 after 4 and $48 \mathrm{~h}$ from 4 independent experiments is shown. Error bars show SEM. ${ }^{*} \mathrm{p}<0.05$.

Phagosome Functionality and

M. tuberculosis Growth fore CD63 staining showed that there was no difference in the proportion of CD63-positive phagosomes between live and dead H37Rv-infected hMDMs (data not shown). Maximal CD63 translocation of about $75-80 \%$ was observed after $4 \mathrm{~h}$ to zymosan- and $\gamma$-irradiated H37Rvcontaining phagosomes. In hMDMs infected with live H37 Rv or H37Ra, however, CD63 translocation was partially inhibited, and only about $40-50 \%$ of the phagosomes were positive for CD63 after $4 \mathrm{~h}$ of infection. This level was similar after 2 days (fig. 2b). Thus, using this assay we confirm that live M. tuberculosis inhibits translocation of CD63 to the phagosome, and show that $\mathrm{H} 37 \mathrm{Rv}$ and H37Ra reduce CD63 translocation to a similar extent.

In order to investigate whether acquisition of CD63 correlated with the intracellular growth restriction observed with $\mathrm{H} 37 \mathrm{Rv}$ at MOI 1, CD63 translocation was quantified in hMDMs infected with $\mathrm{H} 37 \mathrm{Rv}$ or H37Ra at MOI 10 and 1. However, there was no difference between the percentage of CD63-positive phagosomes between MOI 10 and 1 at either time point studied (fig. 2 b), indicating that the macrophages were able to control the infection despite the ineffective translocation of CD63 to the phagosome at the low MOI. It was not possible to compare CD63 translocation beyond 2 days of infection, as there were very few remaining intact hMDMs after $\mathrm{H} 37 \mathrm{Rv}$ infection at MOI 10.

The dominating view is that M. tuberculosis divides inside phagosomes whose communication with lysosomes has been blocked [7]. However, having observed that the lack of CD63 acquisition did not correlate with effective growth of $\mathrm{H} 37 \mathrm{Rv}$ at MOI 1 or H37Ra, we wanted to investigate whether replication of virulent $M$. tuberculosis could also occur inside phagosomes positive for LAMPs. We infected hMDMs with H37Rv at MOI 10 for 4 or $48 \mathrm{~h}$, fixed the cells and stained for the LAMPs CD63 (fig. 2c) and LAMP-1 (fig. 2e). The number of individual bacteria within CD63- or LAMP-1-positive and CD63- or LAMP-1-negative compartments was enumerated, and each bacillus was scored as one event. There was an increase in the average number of CD63- (fig. 2d) or LAMP1-positive (fig. 2f) individual bacteria per hMDM over 2 days (CD63 $\mathrm{p}=0.0101$, LAMP-1 $\mathrm{p}=0.0409$ ). This was probably not merely the result of fusion between phagosomes, as the total number of bacteria in the macrophages increased during this time (fig. 1). Furthermore, while the number of individual bacilli positive for the markers increased over 2 days, the proportion of CD63-positive phagosomes remained the same (fig. 2b). This may indicate that replication of bacilli can occur inside compart-

J Innate Immun 2011;3:508-518 


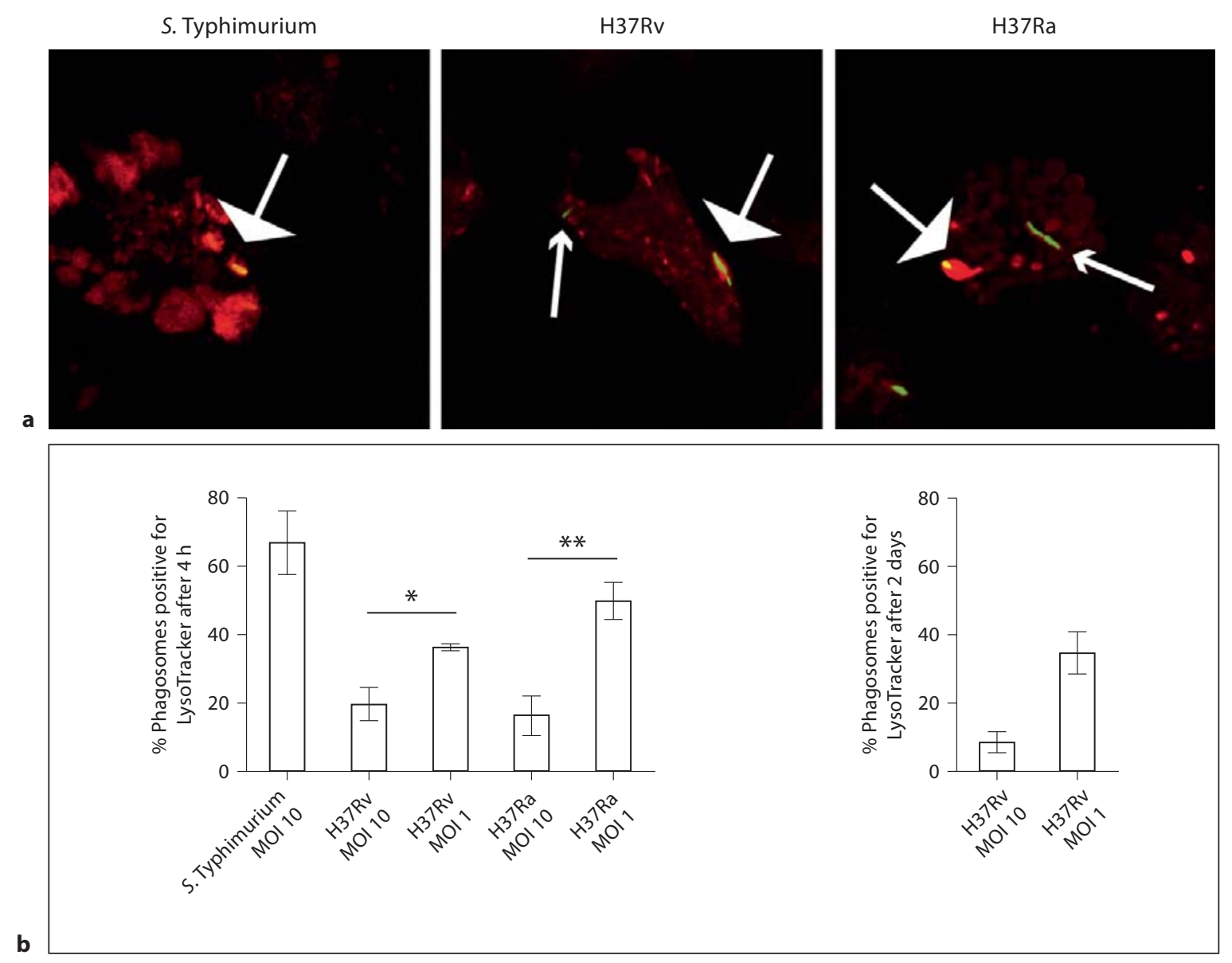

Fig. 3. Acidification of the phagosome after infection with different strains and MOI. hMDMs were infected with GFP-expressing S. Typhimurium, H37Rv or H37Ra at MOI 10 or 1, LysoTracker staining was performed, and the macrophages were fixed at the indicated time points. a Confocal images showing $S$. Typhimurium-, H37Rv- or H37Ra-containing (green) phagosomes stained with LysoTracker (red) after $4 \mathrm{~h}$ of infection. The images are merges of the green and red channels. The large arrows indicate phago- somes positive for LysoTracker, and the small arrows show negative phagosomes. b Quantification of the number of acidified phagosomes in hMDMs challenged with $S$. Typhimurium, H37Rv or H37 Ra at MOI 10 or 1 . The percentage of phagosomes that were positive for LysoTracker after $4 \mathrm{~h}$ and 2 days is shown. The figure depicts the mean and SEM from at least 3 independent experiments (blinded evaluation of $>40$ phagosomes per preparation). ${ }^{*} \mathrm{p}<0.05 ;{ }^{* *} \mathrm{p}<0.01$. ments positive for late endosomal/lysosomal markers as well as in LAMP-negative ones, or possibly that an increasing number of phagosomes form during replication. We conclude that the number of $\mathrm{H} 37 \mathrm{Rv}$ bacteria residing in LAMP-positive compartments in hMDMs increases as the bacteria replicate.

\section{Acidification of the Phagosome Correlates with Growth Restriction at a Low MOI}

The absence of a correlation between inhibition of CD63 translocation and intracellular growth at different MOIs and with different strains prompted us to investigate whether another component of phagosomal matura- tion, acidification, instead correlated with mycobacterial growth restriction. We thus stained macrophages challenged for $4 \mathrm{~h}$ or 2 days with $\mathrm{H} 37 \mathrm{Rv}$ or H37Ra at MOI 10 or 1 with LysoTracker Red, fixed the macrophages and examined the phagosomes using a confocal microscope (fig. 3a). Phagosomes in the images were classified as positive or negative for Lyso Tracker staining. In this assay, single bacilli and aggregates were both scored as one phagosome event, and $S$. Typhimurium-containing hMDMs were used as a control because efficient acidification of $S$. Typhimurium-containing phagosomes has previously been demonstrated [25]. When hMDMs were allowed to phagocytose $S$. Typhimurium at MOI 10, an 


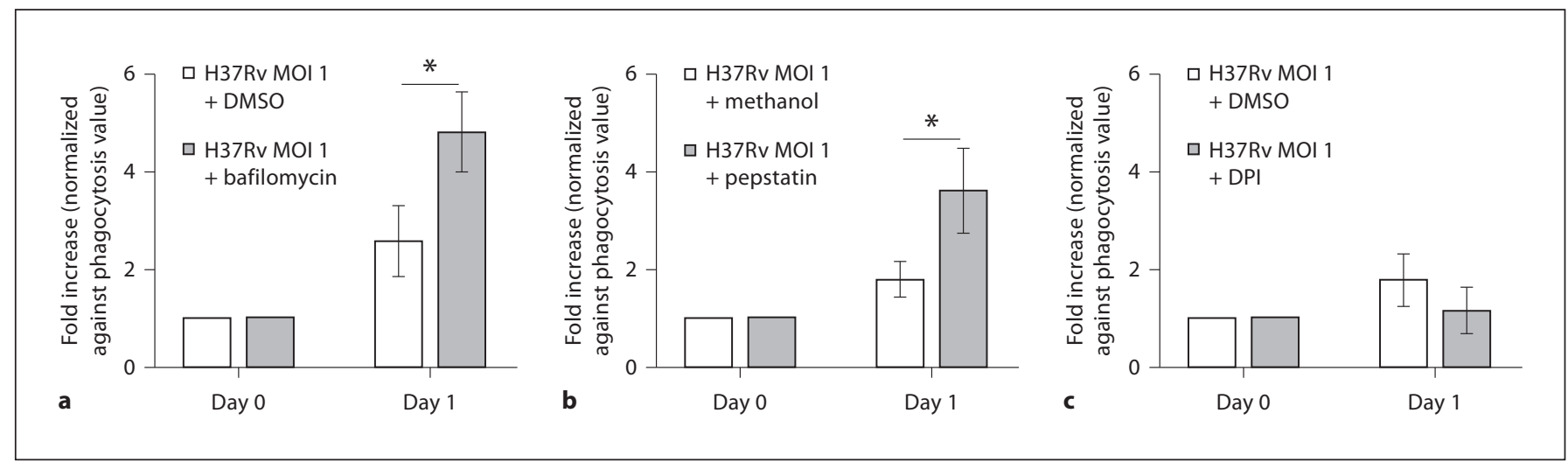

Fig. 4. Replication of H37Rv upon inhibition of phagosomal functions. hMDMs were infected with luciferase-expressing H37Rv at MOI 1 in the presence or absence of different inhibitors and bacterial replication was assessed upon lysis of the hMDMs. The histograms show the fold-increase of H37Rv after $24 \mathrm{~h}$, as compared to the $4 \mathrm{~h}$ value. a The hMDMs were infected in the presence of bafilomycin $(100 \mathrm{nM})$ or DMSO $(0.1 \%)$. b The
hMDMs were infected in the presence of pepstatin $(1 \mu \mathrm{M})$ or methanol $(0.03 \%)$. c The hMDMs were infected in the presence of DPI $(5 \mu \mathrm{M})$ or DMSO (0.1\%). The mean and SEM of 6 (bafilomycin and pepstatin) or 2 (DPI) independent experiments where all values have been normalized against the phagocytosis value are shown. ${ }^{*} \mathrm{p}<0.05$. average of $67 \%$ of phagosomes were positive for LysoTracker after $4 \mathrm{~h}$. This value was reduced in both H37Rvand H37Ra-infected hMDMs to about $20 \%$ at MOI 10 , again confirming that both virulent and avirulent $M$. $t u$ berculosis inhibit phagosomal maturation (fig. 3b). The percentage of phagosomes positive for LysoTracker was significantly higher at MOI 1 than 10 after $4 \mathrm{~h}(\mathrm{H} 37 \mathrm{Rv}$ $\mathrm{p}=0.049, \mathrm{H} 37 \mathrm{Ra} \mathrm{p}=0.008$ ), suggesting more efficient acidification at the lower MOIs (fig. 3b). The inefficient acidification at MOI 10 after $4 \mathrm{~h}$ was not due to cell death as hMDMs infected at both MOIs were viable at this time point, as evidenced by the cell viability assay mentioned above. The difference between MOI 10 and 1 observed at day 2, however, may be due to the extensive cell death at MOI 10 leading to a lack of LysoTracker staining. The loss of hMDM viability also made it difficult to examine whether there was an increase in the number of H37Rv bacilli in LysoTracker-positive and LysoTracker-negative compartments, as studied for LAMPs in figure $2 \mathrm{c}-\mathrm{f}$.

\section{The Vacuolar $H^{+}$-ATPase and Cathepsin D Are \\ Required for Control of H37Rv Growth}

Having established that phagosomal acidification, but not CD63 translocation to the phagosome, correlated with growth inhibition of $\mathrm{H} 37 \mathrm{Rv}$ at the low MOI, we wanted to further characterize the macrophage functions involved in the control of mycobacterial replication. To study the different aspects of phagosomal functionality necessary for the inhibition of mycobacterial growth, we infected hMDMs with H37Rv at MOI 1 and inhibited the vacuolar $\mathrm{H}^{+}$-ATPase using bafilomycin, cathepsin $\mathrm{D}$ using pepstatin, or the NADPH oxidase using DPI, prior to infection and throughout the experiment. The intracellular growth assay showed that addition of bafilomycin (fig. 4a) or pepstatin (fig. 4b) significantly increased the replication efficiency of the bacteria during the first $24 \mathrm{~h}$ of infection as compared to the control (bafilomycin $\mathrm{p}=$ 0.045 , pepstatin $p=0.021$ ). In a control experiment, bafilomycin was able to diminish the number of LysoTracker-positive latex bead phagosomes by $100 \%$ (data not shown), showing that bafilomycin was inhibiting acidification. Addition of DPI, on the other hand, did not affect the growth rate of the bacteria upon infection at MOI 1 (fig. 4c). The results show that the vacuolar $\mathrm{H}^{+}$-ATPase and cathepsin D are important for restricting the intramacrophage growth of $\mathrm{H} 37 \mathrm{Rv}$ at the low MOI.

\section{Discussion}

M. tuberculosis can inhibit the fusion of the phagosome in which it resides with late endosomes and lysosomes, as well as prevent the compartment from acquiring vacuolar $\mathrm{H}^{+}$-ATPases. These events are thought to lead to survival and efficient replication of the bacilli. It is frequently assumed in the literature that since $M$. $t u$ - 
berculosis can inhibit the translocation of late endosomal/lysosomal markers to the phagosome, effective translocation representing phagolysosomal fusion must lead to the killing of the bacilli $[4,26,27]$. However, the relationship between different components of phagosomal maturation and mycobacterial replication in human macrophages has not been extensively studied using virulent $M$. tuberculosis. Here, we investigated which components of phagosomal maturation correlate with inhibition of mycobacterial growth in unstimulated human macrophages, using a low-MOI infection with $\mathrm{H} 37 \mathrm{Rv}$.

By means of a luciferase-based method, we found that human macrophages could control $\mathrm{H} 37 \mathrm{Rv}$ infection at MOI 1, resulting in a balance between the pathogen and the host cell, where there was little net change in bacterial numbers. Conversely, infection at MOI 10 led to effective replication and rapid host cell death. Comparison between different aspects of phagosomal maturation at the two MOIs proved a useful tool in studying which factors are necessary for control of intracellular replication of $M$. tuberculosis. Using a microscopy-based method, we then confirmed that $\mathrm{H} 37 \mathrm{Rv}$ at MOI 10 replicated efficiently inside hMDMs, forming large clusters, while H37Ra did not show any net change in bacterial numbers. $\mathrm{H} 37 \mathrm{Rv}$ but not H37Ra infection at MOI 10 was coupled with decreased hMDM viability over time. Intramacrophage replication of $\mathrm{H} 37 \mathrm{Rv}$ but not $\mathrm{H} 37 \mathrm{Ra}$ is in line with previous studies $[18,22]$, and $\mathrm{H} 37 \mathrm{Ra}$ and $\mathrm{H} 37 \mathrm{Rv}$ have genomic and transcriptional regulation differences which make $\mathrm{H} 37 \mathrm{Rv}$ better equipped for intramacrophage growth [22].

Through staining of CD63, which is a frequently used marker of fusion of phagosomes with late endosomes and lysosomes [3, 28, 29], we found that both the virulent $\mathrm{H} 37 \mathrm{Rv}$ and the avirulent $\mathrm{H} 37 \mathrm{Ra}$ strains were equally able to reduce translocation of late endosomal/lysosomal proteins to the phagosome in comparison to zymosan, consistent with many previous studies $[3,4,27,30]$. This result shows that our assay was capable of detecting differences in CD63 acquisition. The ability to reduce the fusion of the phagosome with late endosomes and lysosomes is thought to lead to protection against microbicidal hydrolases, facilitating replication in immature phagosomes [8]. The association between mycobacterial phagosomes and LAMPs is higher in our study than in another previous report using hMDMs [31], and lower than a third report [21], possibly reflecting differences in macrophage differentiation procedures.

A correlation between the ability to control low-MOI infections and translocation of LAMPs to the phagosome would suggest a major role for fusion of phagosomes with late endosomes/lysosomes in growth restriction at the low MOI. Thus, we studied CD63 translocation to phagosomes containing $\mathrm{H} 37 \mathrm{Rv}$ at a high and low MOI. However, there were no differences in CD63 translocation between the high and low MOIs. This indicates that the ability to restrict acquisition of late endosomal/lysosomal proteins does not in itself mean that growth can occur. Furthermore, we found that as $\mathrm{H} 37 \mathrm{Rv}$ divided inside the macrophages, the number of bacteria in CD63- or LAMP1-positive phagosomes increased, which could indicate that $\mathrm{H} 37 \mathrm{Rv}$ was able to replicate within compartments that have acquired the markers. Replication in phagosomes positive for LAMPs has not previously been analyzed directly, but one study showed that fusion of the mycobacteria-containing vacuole with lysosomes does not lead to bacterial killing, but rather impairment in growth [32]. However, we cannot rule out the alternative explanation that the bacilli have replicated in an unfused phagosome and subsequently been taken up into an autophagosome that has matured [33], resulting in a higher number of LAMP-associated bacilli as well as a higher number of phagosomes over time. Altogether, acquisition of CD63 did not correlate with control of $M$. tuberculosis growth inside the phagosome in our experimental setup. The fact that $M$. tuberculosis avoids phagolysosomal fusion, however, indicates that inhibition of delivery of lysosomal components to the phagosome plays an important role in virulence. Our data rather support the notion that the presence of late endosomal/lysosomal markers, although frequently used to study mycobacterial phagosomes [12], does not completely reflect the environment within the phagosome $[8,9]$. CD63 and other LAMPs are present on intermediate and late endosomes as well as lysosomes, and thus do not reflect a complete maturation state [34].

Besides inhibiting the fusion between phagosomes and lysosomes, $M$. tuberculosis is also able to prevent the vacuolar $\mathrm{H}^{+}$-ATPase from being incorporated into the phagosomal membrane, thus protecting itself from acidification of the vacuole [8-10]. In order to further characterize the mycobacterial phagosomes in our infection model, LysoTracker staining was carried out, and we confirmed again that M. tuberculosis inhibits phagosomal maturation. The fact that $\mathrm{H} 37 \mathrm{Rv}$ and $\mathrm{H} 37 \mathrm{Ra}$ inhibited phagosomal maturation in terms of both CD63 acquisition and acidification to a similar extent despite the difference in intramacrophage growth ability suggests that inhibition of phagosomal maturation is not the mechanism explaining the difference in intracellular replica- 
tion ability observed between $\mathrm{H} 37 \mathrm{Rv}$ and H37Ra, since both strains achieved an equal inhibition of this process. However, our study does not rule out a possible difference related to the sensitivity of the strains to lysosomal contents. The data furthermore revealed that acidification of the phagosome was more efficient at a low MOI, correlating with inhibition of replication. The results obtained with hMDMs infected with $S$. Typhimurium showed that the macrophages were able to handle acidification of many phagosomes in the same hMDM. The ability of $M$. tuberculosis to prevent acidification at higher bacterial loads is therefore not simply an exhaustion of the macrophage capacities, but must involve a specific bacterial mechanism, which is more efficient at high MOI and which results in more efficient reduction of acidification.

Inhibition of the vacuolar $\mathrm{H}^{+}$-ATPase and assessment of bacterial replication showed that lack of phagosomal acidification increased the replication efficiency of $\mathrm{H} 37 \mathrm{Rv}$, confirming data obtained with avirulent mycobacteria [35]. We suggest a major role for acidification in limiting growth of virulent $M$. tuberculosis inside human macrophages, which may be direct or indirect, consistent with previous studies $[10,11]$. One study showed that $M$. tuberculosis can survive acidic environments and suggested that inhibition of acidification is a strategy for avoiding other phagolysosomal functions [36], as acidification of the phagosome is important for the function of degradative lysosomal enzymes and the generation of hydrogen peroxide in the phagosome [37]. The fact that cathepsin D was also necessary for limitation of bacterial growth at MOI 1 suggests that acidification may indirectly contribute to the growth restriction. However, our data did not reveal a role for the NADPH oxidase and thus reactive oxygen intermediates in controlling mycobacterial growth. As reviewed by Huynh and Grinstein [37], the relationship between phagolysosomal fusion and phagosomal acidification is complex, since acidification is sometimes regarded as a determinant of phagosomal maturation, with a role in endosomal trafficking, and sometimes as a consequence, dependent on fusion with lysosomes. The mechanism by which the vacuolar $\mathrm{H}^{+}$ATPase is recruited to the phagosome is also not completely clear, but previous studies suggest trafficking from the trans-Golgi network $[38,39]$ or delivery from endosomes or lysosomes [35, 40]. Phagolysosomal fusion, on the other hand, is dependent on recruitment of the organelle tethering molecule early endosomal antigen 1 [12]. It was clear from our data that CD63 acquisition and phagosomal acidification occurred at different rates, especially at MOI 1 , which makes us speculate that the vac- uolar $\mathrm{H}^{+}$-ATPase and late endosomal/lysosomal proteins are acquired by different routes. A previous study showed that CD63 translocation occurred at similar levels to phagosomes containing both metabolically active and inactive $M$. tuberculosis, while LysoTracker only associated with inactive bacilli [21]. This is consistent with the notion that LAMP translocation does not truly reflect the phagosomal environment, but that acidification is a better marker for phagosome function.

In conclusion, we found that inhibition of CD63 translocation to the phagosome did not per se lead to effective mycobacterial growth inside the macrophage. On the other hand, phagosomal acidification and functional cathepsin D played important roles in growth restriction of virulent $M$. tuberculosis at a low MOI in unstimulated human macrophages. The importance of macrophage functionality in vivo should be the focus of future studies, to establish what factor or factors are critical for growth arrest of $M$. tuberculosis during natural infection.

\section{Acknowledgements}

We are grateful to Lalita Ramakrishnan for providing the pFPV2 plasmid and to Douglas Young for providing the pSMT1 plasmid.

This work was supported by grants from the Swedish Research Council (No. 529-2003-5994, 2005-7046, 2006-5968, 2007-2673, 2009-3821), Bill and Melinda Gates Foundation, SIDA/SAREC, Ekhaga Foundation, Carl Trygger Foundation, King Gustaf V 80Year Memorial Foundation, County Council of Östergötland, Swedish Heart Lung Foundation, Oskar II Jubilee Foundation, Clas Groschinsky Foundation and Söderbergs Foundation. $\gamma$-Irradiated $M$. tuberculosis was provided by Colorado State University, Fort Collins (NIH, NIAID contract No. HHSN26620040 0091C, entitled 'Tuberculosis Vaccine Testing and Research Materials').
References
1 Scherr N, Jayachandran R, Mueller P, Pieters $\mathrm{J}$ : Interference of Mycobacterium tuberculo- sis with macrophage responses. Indian J Exp Biol 2009;47:401-406.
2 Armstrong JA, Hart PD: Response of cul- tured macrophages to Mycobacterium tuber- culosis, with observations on fusion of lyso- somes with phagosomes. J Exp Med 1971; 134:713-740.
-3 Clemens DL, Horwitz MA: Characterization of the Mycobacterium tuberculosis phago- some and evidence that phagosomal matura- tion is inhibited. J Exp Med 1995;181:257- 270.
4 Russell DG: Mycobacterium tuberculosis: here today, and here tomorrow. Nat Rev Mol Cell Biol 2001;2:569-577. 
-5 Via LE, Deretic D, Ulmer RJ, Hibler NS, Huber LA, Deretic V: Arrest of mycobacterial phagosome maturation is caused by a block in vesicle fusion between stages controlled by rab5 and rab7. J Biol Chem 1997;272:13326-13331.

-6 van der Wel N, Hava D, Houben D, Fluitsma D, van Zon M, Pierson J, Brenner M, Peters PJ: M. Tuberculosis and M. leprae translocate from the phagolysosome to the cytosol in myeloid cells. Cell 2007;129:1287-1298.

7 Meena LS, Rajni: Survival mechanisms of pathogenic Mycobacterium tuberculosis H37Rv. Febs J 2010;277:2416-2427.

8 Rohde K, Yates RM, Purdy GE, Russell DG: Mycobacterium tuberculosis and the environment within the phagosome. Immunol Rev 2007;219:37-54.

-9 Sturgill-Koszycki S, Schlesinger PH, Chakraborty P, Haddix PL, Collins HL, Fok AK, Allen RD, Gluck SL, Heuser J, Russell DG: Lack of acidification in Mycobacterium phagosomes produced by exclusion of the vesicular proton-atpase. Science 1994;263: 678-681.

-10 Stewart GR, Patel J, Robertson BD, Rae A, Young DB: Mycobacterial mutants with defective control of phagosomal acidification. PLoS Pathog 2005;1:269-278.

- 11 Schaible UE, Sturgill-Koszycki S, Schlesinger PH, Russell DG: Cytokine activation leads to acidification and increases maturation of Mycobacterium avium-containing phagosomes in murine macrophages. J Immunol 1998;160:1290-1296.

12 Vergne I, Chua J, Singh SB, Deretic V: Cell biology of Mycobacterium tuberculosis phagosome. Annu Rev Cell Dev Biol 2004; 20:367-394.

$\checkmark 13$ Pethe K, Swenson DL, Alonso S, Anderson J, Wang C, Russell DG: Isolation of Mycobacterium tuberculosis mutants defective in the arrest of phagosome maturation. Proc Natl Acad Sci USA 2004;101:13642-13647.

- 14 Thoma-Uszynski S, Stenger S, Takeuchi O, Ochoa MT, Engele M, Sieling PA, Barnes PF, Rollinghoff M, Bolcskei PL, Wagner M, Akira S, Norgard MV, Belisle JT, Godowski PJ, Bloom BR, Modlin RL: Induction of direct antimicrobial activity through mammalian Toll-like receptors. Science 2001;291:15441547.

15 Reljic R, Stylianou E, Balu S, Ma JK: Cytokine interactions that determine the outcome of mycobacterial infection of macrophages. Cytokine 2010;51:42-46.

- 16 Valdivia RH, Hromockyj AE, Monack D, Ramakrishnan L, Falkow S: Applications for green fluorescent protein (GFP) in the study of host-pathogen interactions. Gene 1996; 173:47-52.
17 Welin A, Winberg ME, Abdalla H, Sarndahl E, Rasmusson B, Stendahl O, Lerm M: Incorporation of Mycobacterium tuberculosis lipoarabinomannan into macrophage membrane rafts is a prerequisite for the phagosomal maturation block. Infect Immun 2008;76:2882-2887.

18 Eklund D, Welin A, Schon T, Stendahl O, Huygen K, Lerm M: Validation of a mediumthroughput method for evaluation of intracellular growth of Mycobacterium tuberculosis. Clin Vaccine Immunol 2010;17:513-517.

19 Fadok VA, Bratton DL, Konowal A, Freed PW, Westcott JY, Henson PM: Macrophages that have ingested apoptotic cells in vitro inhibit pro-inflammatory cytokine production through autocrine/paracrine mechanisms involving TGF- $\beta$, PGE2, and PAF. Clin Invest 1998;101:890-898.

20 Savill JS, Wyllie AH, Henson JE, Walport MJ, Henson PM, Haslett C: Macrophage phagocytosis of aging neutrophils in inflammation. Programmed cell death in the neutrophil leads to its recognition by macrophages. J Clin Invest 1989;83:865-875.

21 Lee BY, Clemens DL, Horwitz MA: The metabolic activity of Mycobacterium tuberculosis, assessed by use of a novel inducible GFP expression system, correlates with its capacity to inhibit phagosomal maturation and acidification in human macrophages. Mol Microbiol 2008;68:1047-1060.

22 Lee JS, Krause R, Schreiber J, Mollenkopf HJ, Kowall J, Stein R, Jeon BY, Kwak JY, Song MK, Patron JP, Jorg S, Roh K, Cho SN, Kaufmann SH: Mutation in the transcriptional regulator PHOP contributes to avirulence of Mycobacterium tuberculosis H37Ra strain. Cell Host Microbe 2008;3:97-103.

23 Sturgill-Koszycki S, Schaible UE, Russell DG: Mycobacterium-containing phagosomes are accessible to early endosomes and reflect a transitional state in normal phagosome biogenesis. EMBO J 1996;15:69606968

24 Ullrich HJ, Beatty WL, Russell DG: Direct delivery of procathepsin $\mathrm{d}$ to phagosomes: implications for phagosome biogenesis and parasitism by Mycobacterium. Eur J Cell Biol 1999;78:739-748.

25 Rathman M, Sjaastad MD, Falkow S: Acidification of phagosomes containing Salmonella typhimurium in murine macrophages. Infect Immun 1996;64:2765-2773.

-26 Kusner DJ: Mechanisms of mycobacterial persistence in tuberculosis. Clin Immunol 2005; 114:239-247.

27 de Chastellier C: The many niches and strategies used by pathogenic mycobacteria for survival within host macrophages. Immunobiology 2009;214:526-542.
28 Malik ZA, Thompson CR, Hashimi S, Porter B, Iyer SS, Kusner DJ: Cutting edge: Mycobacterium tuberculosis blocks $\mathrm{Ca}^{2+}$ signaling and phagosome maturation in human macrophages via specific inhibition of sphingosine kinase. J Immunol 2003;170:2811-2815.

-29 Fratti RA, Chua J, Deretic V: Induction of p38 mitogen-activated protein kinase reduces early endosome autoantigen 1 (EEA1) recruitment to phagosomal membranes. J Biol Chem 2003;278:46961-46967.

30 O'Leary S, O'Sullivan MP, Keane J: IL-10 blocks phagosome maturation in Mycobacterium tuberculosis-infected human macrophages. Am J Respir Cell Mol Biol DOI: 10.1165/rcmb.2010-0319OC [Epub ahead of print].

31 Harris J, Hope JC, Keane J: Tumor necrosis factor blockers influence macrophage responses to Mycobacterium tuberculosis. J Infect Dis 2008;198:1842-1850.

32 Gomes MS, Paul S, Moreira AL, Appelberg R, Rabinovitch M, Kaplan G: Survival of $M y$ cobacterium avium and Mycobacterium tuberculosis in acidified vacuoles of murine macrophages. Infect Immun 1999;67:31993206.

33 Deretic V: Autophagy, an immunologic magic bullet: Mycobacterium tuberculosis phagosome maturation block and how to bypass it. Future Microbiol 2008;3:517-524.

-34 Flannagan RS, Cosio G, Grinstein S: Antimicrobial mechanisms of phagocytes and bacterial evasion strategies. Nat Rev Microbiol 2009;7:355-366.

- 35 Jordao L, Bleck CK, Mayorga L, Griffiths G, Anes E: On the killing of mycobacteria by macrophages. Cell Microbiol 2008;10:529548.

36 Vandal OH, Pierini LM, Schnappinger D, Nathan CF, Ehrt S: A membrane protein preserves intrabacterial $\mathrm{pH}$ in intraphagosomal Mycobacterium tuberculosis. Nat Med 2008; 14:849-854.

37 Huynh KK, Grinstein S: Regulation of vacuolar $\mathrm{pH}$ and its modulation by some microbial species. Microbiol Mol Biol Rev 2007;71: 452-462.

38 Fratti RA, Chua J, Vergne I, Deretic V: Mycobacterium tuberculosis glycosylated phosphatidylinositol causes phagosome maturation arrest. Proc Natl Acad Sci USA 2003; 100:5437-5442.

39 Kinchen JM, Ravichandran KS: Phagosome maturation: going through the acid test. Nat Rev Mol Cell Biol 2008;9:781-795.

-40 Sun-Wada GH, Tabata H, Kawamura N, Aoyama M, Wada Y: Direct recruitment of $\mathrm{H}+$-ATPase from lysosomes for phagosomal acidification. J Cell Sci 2009;122:25042513. 